\title{
Detection of Disbonds in Multi-layer Structures by Laser-Based Ultrasonic Technique
}

\author{
D. Cerniglia, N. Montinaro, and V. Nigrelli \\ Dipartimento di Meccanica, University of Palermo, Viale delle \\ Scienze, Palermo, Italy
}

\begin{abstract}
Adhesively bonded multi-layer structures are frequently used, mostly in the aerospace industry, for their structural efficiency. Nondestructive evaluation of bond integrity in these types of structures, both after manufacturing and for periodic inspection during service, is extremely important.
\end{abstract}

A laser-based ultrasonic technique has been evaluated for non-contact detection of disbonds in aluminum multi-layer structures. Two configurations have been used to detect disbonded areas: pitch-catch with unidirectional guided wave scan and through-transmission with bidirectional scan. Guided wave scanning was done with a laser line source and air-coupled transducer sensing at $500 \mathrm{kHz}, 1 \mathrm{MHz}$, and $2 \mathrm{MHz}$. Signals showed attenuation of the main frequency component and frequency shift on disbonded areas, whereas, a regular and standard waveform is seen outside disbonds. In through-transmission the longitudinal wave at normal incidence was monitored with a $1 \mathrm{MHz}$ probe. One sample showed, besides the introduced inserts, other disbonded areas. After the ultrasonic measurements the sample was cut to visually check adhesive and interfaces.

The guided wave pitch-catch scan allowed fast inspection and quick indication of disbonded zones, while the through-transmission C-Scan provided better definition of defects but was slower and required access from both sides of the test part.

Keywords: Guided waves; Laser ultrasound; Multi-layer structures

\section{INTRODUCTION}

Adhesively bonded multi-layer structures are of interest in many industries, especially in aerospace where bonded aluminum and composite structures are widely used in primary and secondary structures of aircraft [1,2]. Major aircraft manufacturers use significant amounts

Received 11 February 2008; in final form 24 July 2008.

Address correspondence to Donatella Cerniglia, Dipartimento di Meccanica, University of Palermo, Viale delle Scienze, Palermo, Italy. E-mail: cerniglia@dima.unipa.it 
of new materials (composites and aluminum alloys) to achieve lighter weight. Lighter aircrafts mean less fuel that is burned; so reducing weight is also important in reducing $\mathrm{CO}_{2}$ emissions. Even when content of composite materials in aircraft has become significant over the years (e.g., being used on fuselage, wings, tail, doors, and interior), aluminum still remains a remarkably useful material for such structures (e.g., used on wing and tail leading edges) and better aluminum alloys have been developed.

Besides aerospace, many other industries use adhesive bonding to manufacture and repair engineering structures (marine, offshore, etc.) [3]. Specific advantages of adhesives are a rather easy manufacturing and economic assembly, even distribution of mechanical loads, low weight, no heavy screws and bolts, high stiffness, and decreased stress concentration. A potential limitation is that long term behavior is not fully understood.

The integrity and reliability of the bonds in multi-layer structures are extremely important to the quality of the product. Defects in adhesive bonds can be inside the adhesive layer, due to inclusions or voids, normally affecting the strength of the bulk adhesive. They may also occur at the adherent-adhesive interface, in which case there is no contact or a weak bond between adherent and adhesive, which will produce a disbond under a certain load. Furthermore, bonded multi-layer structure strength can be compromised due to adhesive aging and decay of properties affected by environmental conditions.

The wide range of materials and configurations used in the multilayered structures and the need for nondestructive evaluation (NDE) of these bonds have resulted in different testing methods. However, no single NDE technique has been found adequate to inspect and evaluate the wide variety of applications. Major test methods used to inspect for defects in the range of materials utilized in adhesive-bonded joints are radiographic, based on thermal principles and ultrasonic [4,5].

Conventional ultrasonic NDE techniques are mostly based on contact methods; they cannot be used over irregular or difficult to access surfaces and require smoothness of the test object surface to guarantee proper coupling, to facilitate scanning with the probes, and to produce reliable results. Immersion or water-jet techniques are considered as the most effective because they provide efficient and relatively uniform coupling between the probes and the test piece. Conventional longitudinal wave, shear wave, and high power ultrasonic techniques have been used in Reference [6] to detect dry kissing bonds in adhesive joints. The sensitivity of adhesive bond properties to guided waves, excited and received with broadband immersion probes, has been analyzed in Reference [7] in a three-layer model. 
In the last years non-contact ultrasound within the NDE methods had further development thanks to innovative probes, made with new materials for acoustic devices and manufacturing technologies $[8,9]$. The use of a laser to generate acoustic waves is also an emerging approach, advantageous as inspection is remote, does not depend on contact conditions (pressure, coupling medium, contact area), allows development of automated systems, and operation in hostile environment is feasible by guiding the laser beam through proper delivery optics [10-12].

There has been extensive research directed to characterize adhesively bonded structures using non-contact ultrasonic methods. Air-coupled wideband capacitance transducers, at frequencies below $2 \mathrm{MHz}$, have been used in Reference [13] for non-contact measurements of material properties in adhesively bonded multi-layer structures. High accuracy of measurements is shown by detecting $\sim 30 \mu \mathrm{m}$ thickness variation in a lap-joint.

Ultrasonic inspection of aerospace composite components has been conducted by Hsu [14] using air-coupled piezoelectric transducers at frequencies of 120 and $200 \mathrm{kHz}$ in through-transmission C-Scan mode with a manual scan system. The main difficulty with air-coupled generation and detection is the extremely inefficient energy transfer at the air/solid interface; only about $0.04 \%$ of incident energy is transmitted at the air-carbon composite interface.

Laser ultrasonic techniques together with the 2D Fourier transform are used in Reference [15] to characterize adhesive bond properties. Transient Lamb waves are measured in two types of bonded Al plates to determine the influence of the condition of the adhesive material on the dispersion curves.

In this paper, detection of disbonds in multi-layer aluminum samples with simulated disbonded areas has been performed using a laser/air-coupled ultrasonic system, performing first an L-Scan in pitch-catch configuration and then a C-Scan in through-transmission configuration. The first allowed a quick inspection (scanning is only in one direction since Lamb waves propagate through the whole thickness) but only one dimension of disbonded area was determined; the second method was slower, required access on both sides of the sample, but was more precise in defining shape and size of defects.

\section{THEORETICAL BACKGROUND}

Lamb waves propagate in thin plate-like structures, guided by the plate boundaries, depending on properties of the structure as well as on the thickness and on the vibrational frequency of the wave 
[16,17]; phase and group velocity of each mode is a function, for a given material, of the product between ultrasonic frequency $(f)$ and plate thickness $(d)$. The particle displacement pattern across the thickness is different for the different Lamb modes (symmetric and antisymmetric) and for the same mode changes with the $f \cdot d$ product. For a single-layered isotropic structure, the Lamb wave dispersion curves can be easily plotted from the Rayleigh-Lamb dispersion equations [18]. Propagation of guided waves in multi-layered plates requires a numerical approach, where dispersion curve characteristics depend on the combination of the material properties and thickness of each layer.

Theoretical procedures (e.g., cut-off frequency, frequency compensation method, etc.) allow one to quantitatively determine thickness variation; it becomes more complex to assess adhesive aging or quality of the bond at the adhesive/adherent interface in a multi-layer structure. Variations in the guided wave propagation characteristics (frequency or velocity) can be used to assess changes in the integrity of the structure, as each mode propagates with its displacement pattern at a certain value of the frequency thickness product (as given in the dispersion curves). In fact, frequency or velocity variation will cause a change of the point on the dispersion curves which, in turn, will result (from Snell's law) in a variation of the transmitting angle. If the orientation of the receiving transducer does not change, the final effect will be the amplitude attenuation of the acquired signal.

\section{EXPERIMENTAL PROCEDURE}

\subsection{Bonded Multi-layer Samples}

Adhesive bonding requires competence in material science (substrate and adhesive), pre-treatments, and surface characterization. A clean, dry surface is a necessary prerequisite for achieving good adhesive bonding together with proper surface wetting and curing of the adhesive. Important variables for applying and distributing the adhesive of the substrate are adhesive viscosity and the chemical resistance of the substrate to the adhesive. Poor bonding occurs when the adhesive layer does not stick properly to the substrate; that is why pre-treatment of the surface is helpful. In fact, porous materials are simple to bond to, provided they are dry, whereas non-porous surfaces, as found on metal, should be degreased, abraded, and degreased again. For best results, surfaces can be degreased and chemically pre-treated.

Two samples have been manufactured by bonding together three layers in $\mathrm{Al} 7075-\mathrm{T} 6$, with size $140 \times 180 \mathrm{~mm}$ and thickness (in $\mathrm{mm}$ ) 
TABLE 1 Properties and Performance Characteristics (at $23 \pm 2{ }^{\circ} \mathrm{C}$ ) of the Epoxy Adhesive

\begin{tabular}{lc}
\hline Adhesive type & $\begin{array}{c}\text { Epoxy, two-part room } \\
\text { temperature curing adhesive }\end{array}$ \\
\hline Volume mix ratio (base:accelerator) & $2: 1$ \\
Viscosity & Non-sagging paste \\
Average overlap shear strength & $29.2 \mathrm{MPa}$ \\
Compression strength & $78.8 \mathrm{MPa}$ \\
Young's moduli & $5972 \mathrm{MPa}$ \\
\hline
\end{tabular}

2/2/2 (Sample \#1) and 1/1/2 (Sample \#2), with a bi-component epoxy resin whose properties are reported in Table 1. Particular care was used during manufacturing to completely and uniformly impregnate the surface of the Al plates, after abrading and degreasing with a solvent. The application of the adhesive on the surfaces has been performed differently for the two samples: on \#1 the adhesive has been spread on the surface by the mixer nozzle and naturally distributed by the load pressure above the sample. On Sample \#2 the distribution of the adhesive has been completed using a spatula, before applying the load. The first method has proved to be less effective due to air bubbles trapped at the interface. Thickness of the two adhesive layers is quite uniform across each sample, as seen after cross-cutting the samples, being around $0.3 \mathrm{~mm}$ in Sample \#1 and $0.45 \mathrm{~mm}$ in Sample \#2 for each layer.

Teflon ${ }^{\circledR}$ inserts have been introduced to simulate disbonds at both adhesive layers for each sample, as indicated in Figures 1 and 2. Sample \#1 has four inserts at the first adhesive layer, two are 10 by $10 \mathrm{~mm}$ (A1 and B) and two are 5 by $10 \mathrm{~mm}$ (C and D1) ( $\mathrm{x}$ and y directions, respectively), spaced $30 \mathrm{~mm}$ along the $\mathrm{y}$-direction from center to center. At the second adhesive layer there are two defects, 10 by $10 \mathrm{~mm}$ (A2) and 5 by $10 \mathrm{~mm}$ (D2), corresponding to the same position of inserts A1 and D1 at the first interface. Similarly, Sample \#2 has four inserts at the first adhesive layer, two are 30 by $10 \mathrm{~mm}$ (E1 and $\mathrm{F}$ ) and two are 20 by $10 \mathrm{~mm}$ ( $\mathrm{G}$ and $\mathrm{H} 1$ ) (x and y directions, respectively), spaced $30 \mathrm{~mm}$ along the y-direction from center to center. At the second adhesive layer, there are two defects, 30 by $10 \mathrm{~mm}$ (E2) and 20 by $10 \mathrm{~mm}$ (H2), corresponding to the same position of inserts $\mathrm{E} 1$ and $\mathrm{H} 1$ at the first interface. Details of the samples, the layers, and the Teflon inserts are given in Table 2. 


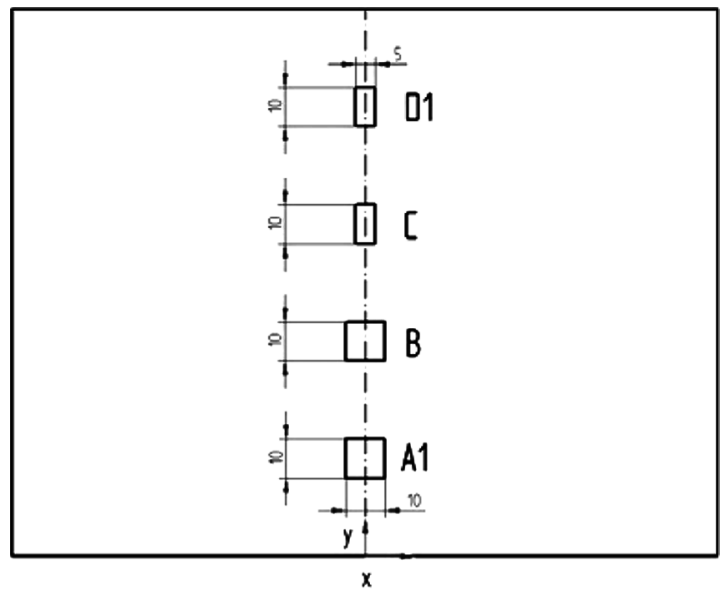

(a)

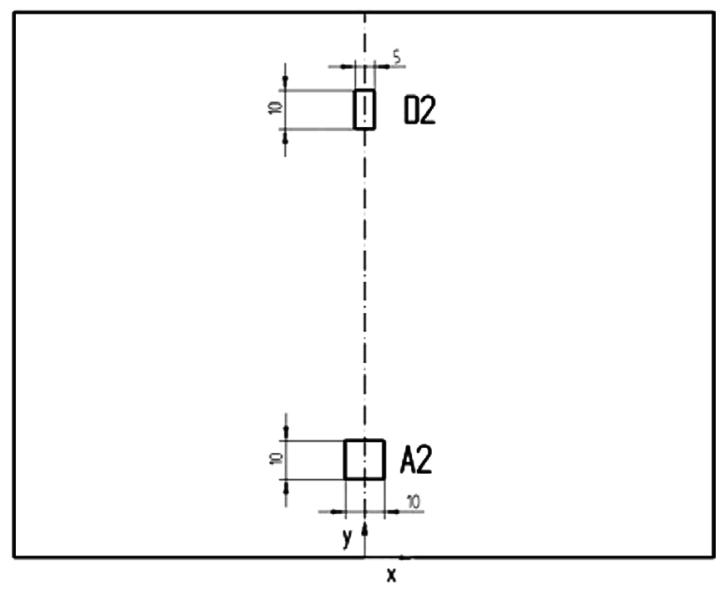

(b)

FIGURE 1 Layout, position, and size (in scale) of defects inserted in Sample $\# 1$ at the (a) first and at the (b) second adhesive layer.

\subsection{Ultrasonic System}

Inspection of samples has been performed in L-Scan pitch-catch configuration (Figure 3a) and in C-Scan through-transmission configuration (Figure 3b). The pulsed laser, Brilliant B, frequency $10 \mathrm{~Hz}$, max energy $900 \mathrm{~mJ}$, wavelength $1064 \mathrm{~nm}$ (Quantel, Paris, France), with $\sim 6 \mathrm{nsec}$ pulse duration, was used at $250 \mathrm{~mJ}$ with the laser beam focused to a $9 \times 1 \mathrm{~mm}$ line source in pitch-catch configuration and to a 


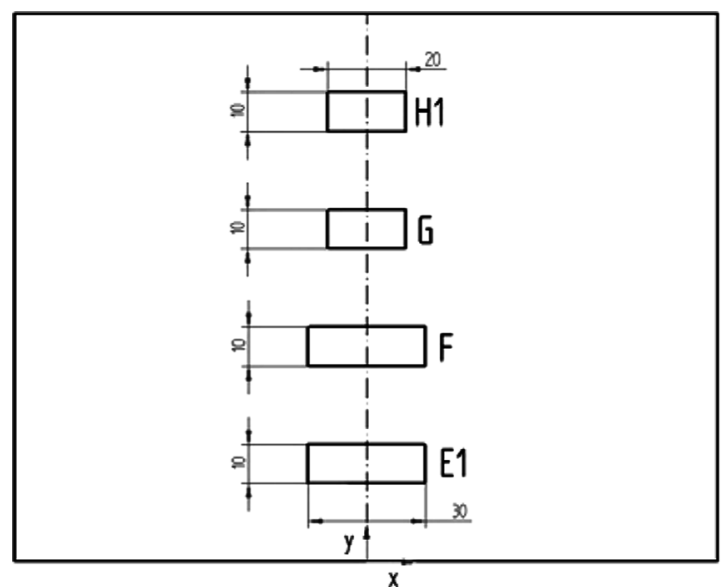

(a)

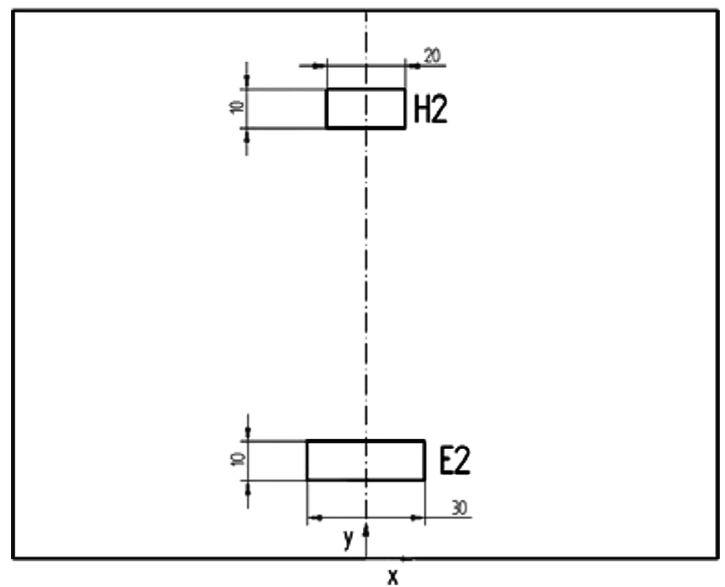

(b)

FIGURE 2 Layout, position, and size (in scale) of defects inserted in Sample \#2 at the (a) first and at the (b) second adhesive layer.

$2 \mathrm{~mm}$ point source in through-transmission. Line source was used to give more directionality to the guided waves, perpendicularly to the line, thus optimizing the use of laser energy (otherwise propagating in all directions with a point source) and, at the same time, improving detection with an air-coupled transducer, which is important as air is a very poor ultrasonic couplant due to the large acoustic impedance $(Z)$ mismatch at the aluminum/air interface $\left(\mathrm{Z}_{\mathrm{Al}} \approx\right.$ $17 \times 10^{6} \mathrm{~kg} / \mathrm{m}^{2} \mathrm{~s}, \mathrm{Z}_{\text {air }} \approx 400 \mathrm{~kg} / \mathrm{m}^{2} \mathrm{~s}$ ) where almost $100 \%$ of the ultrasonic energy will be reflected. 


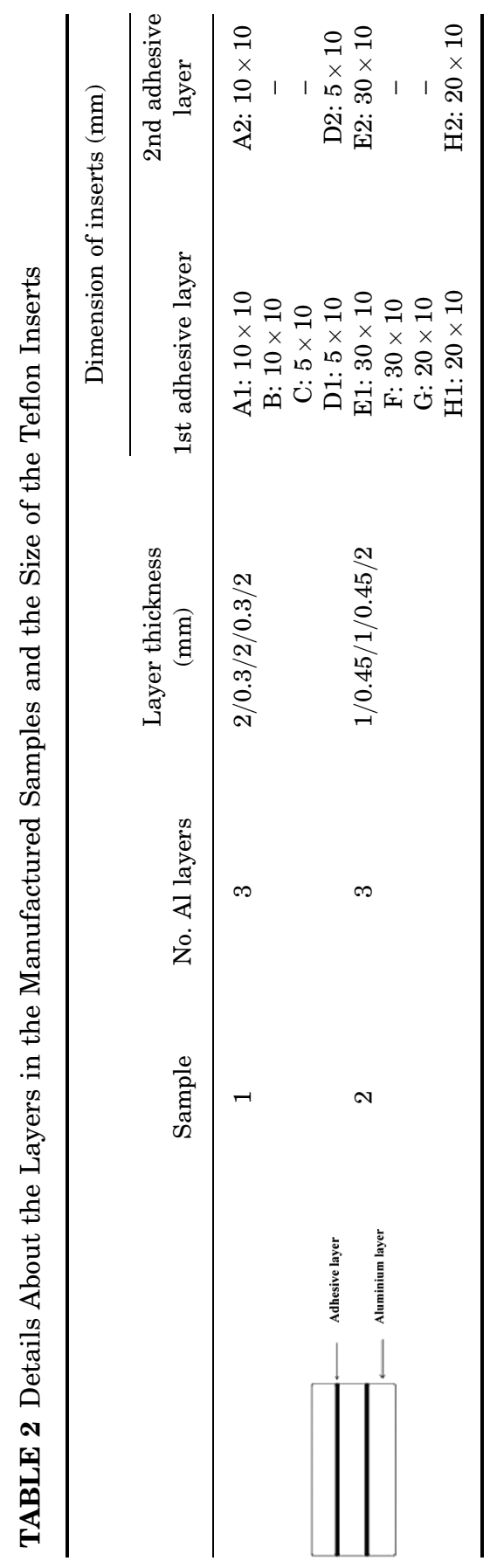




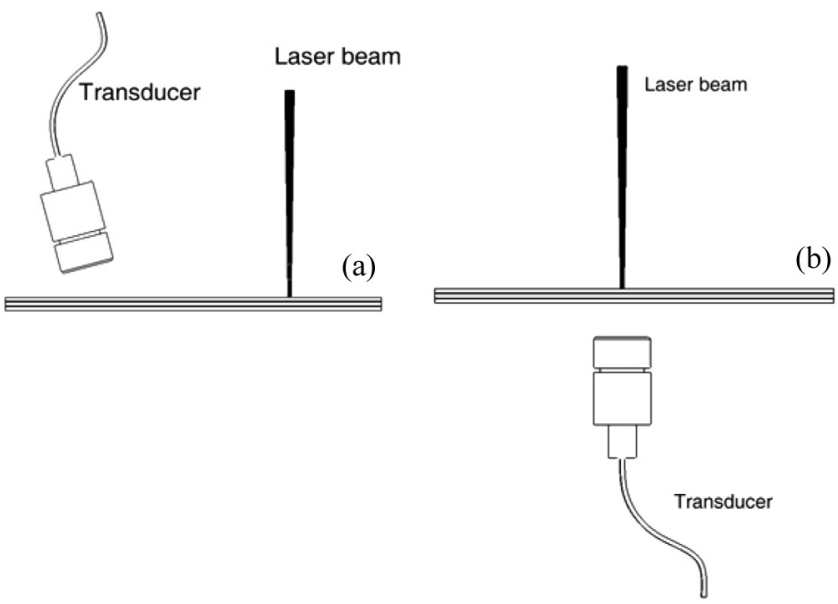

FIGURE 3 Schematic representation of the two experimental setups: (a) pitchcatch and (b) through-transmission configuration.

Description of mechanisms and patterns for ultrasonic waves generated by a laser source (point, line, and array) in the three different regimes (thermoelastic, ablative, and constrained surface) can be found in References [10,19,20]. The use of an array of laser sources to generate narrow-band ultrasonic signals and phased array single pulses, with a significant increase in the sensitivity, is shown in References [21,22].

Since the frequency content of laser-generated Lamb waves depends on the reciprocal of the laser pulse width the spectrum is broad band, inducing various modes that overlap in time domain. Moreover, the presence of more than one adhesive layer determines complex waveforms as the waves reflected from each interface interfere with each other, making mode identification a challenging task. Typically, for pulses of tens of nsec most of the spectrum energy is delivered at frequencies below $50 \mathrm{MHz}$. Thus, while the signal spectrum produced by the $6 \mathrm{nsec}$ laser pulse is fairly broadband $(>100 \mathrm{MHz}$ ), the frequency content of the acquired signals is narrowed by the ultrasonic frequency response of the air-coupled transducers. In fact, they act like a band-pass filter, selecting only wave modes within that frequency range, that are further reduced with transducer orientation. Air-coupled ultrasonic transducers used for testing, with $12.5 \mathrm{~mm}$ diameter of the active area, have bandwidth (at $-6 \mathrm{~dB}$ ) of $130 \mathrm{kHz}$ for the $500 \mathrm{kHz}, 455 \mathrm{kHz}$ for the $1 \mathrm{MHz}$, and $240 \mathrm{kHz}$ for the $2 \mathrm{MHz}$ probe (Ultran, State College, PA, USA). 


\subsection{L-Scan Test}

Uni-directional scanning in pitch-catch configuration was done in $5 \mathrm{~mm}$ steps, which allowed a partial overlapping of laser shots with a line source of $9 \mathrm{~mm}$; source-to-receiver distance (i.e., the inspected region) was $50 \mathrm{~mm}$, with the transducer oriented at $\sim 6^{\circ} \mathrm{C}$ with respect to the normal to the plate. Air-coupled sensing was conducted at $500 \mathrm{kHz}, 1 \mathrm{MHz}$, and $2 \mathrm{MHz}$, high-pass filtering the signal at $80 \mathrm{kHz}$ before amplification.

Longitudinal and surface waves are excited in the plate where the laser beam is directed. After multiple reflections (according to Snell's law) and mode conversions at each interface, interference will lead to the formation of Lamb modes that will depend on the number and sequence of layers, and on their material and thickness. As a result, the Teflon insert is expected to cause the presence of a different converted or attenuated mode (for a certain source-to-receiver distance), even though the effect of real adhesive disbonds will be more evident since the impedance mismatch between epoxy/air/Al is higher than between epoxy/Teflon/Al (see Table 3).

Time domain waveforms acquired in Sample \#1 with 1 and $2 \mathrm{MHz}$ probes are shown in Figures 4 and 5, respectively, for a well-bonded area (left) and for the zone with inserts D1/D2 (right). Time windowing was used to cut off reflections from plate edges.

Signals from Sample \#1, acquired with the $1 \mathrm{MHz}$ probe, showed attenuation of the main frequency component and frequency shift on disbonded areas but also between defects $\mathrm{A}$ and $\mathrm{B}$, and $\mathrm{B}$ and $\mathrm{C}$. Figure 6 shows the Fast Fourier Transform (FFT) of signals acquired in a well-bonded region (between defects $\mathrm{C}$ and $\mathrm{D}$ ) and at defects $\mathrm{A} 1 / \mathrm{A} 2, \mathrm{~B}, \mathrm{C}$, and D1/D2. All the FFT spectra are normalized by dividing the amplitudes of the components by $\mathrm{N} / 2$, where $\mathrm{N}$ is the number of data points (10000 for the considered signals). Frequency response (within the bandwidth of the transducer) provided good information

TABLE 3 Velocity of Longitudinal Wave and Acoustic Impedance for Some Materials

\begin{tabular}{lcc}
\hline Material & $\begin{array}{c}\text { Velocity of longitudinal } \\
\text { wave }(\mathrm{mm} / \mu \mathrm{s})\end{array}$ & $\begin{array}{c}\text { Acoustic impedance } \\
\left(10^{6} \cdot \mathrm{kg} \cdot \mathrm{m}^{-2} \mathrm{~s}^{-1}\right)\end{array}$ \\
\hline Aluminum & 6.32 & 17.1 \\
Epoxy resin & 2.9 & 3.6 \\
Teflon & 1.35 & 2.97 \\
Air & 0.34 & 0.0004 \\
\hline
\end{tabular}




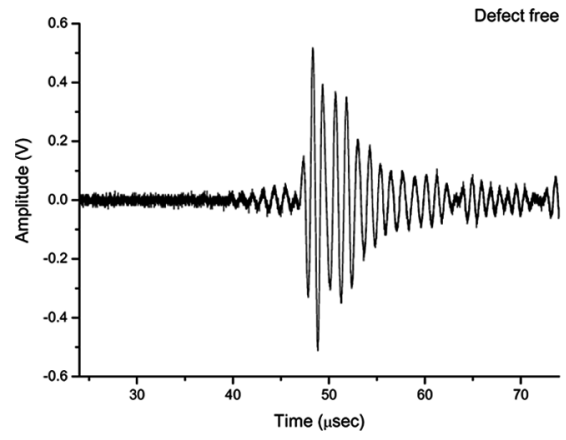

(a)

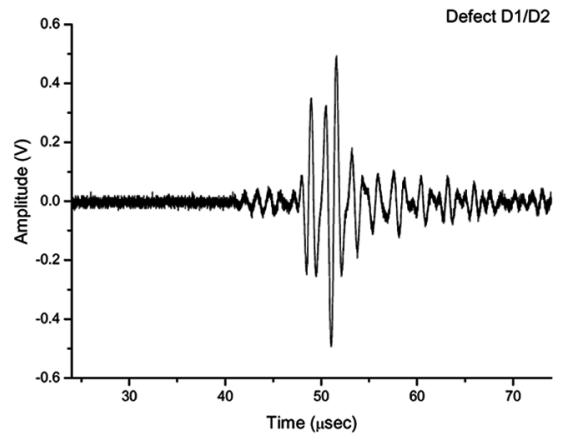

(b)

FIGURE 4 Time domain waveforms acquired in Sample \#1 in pitch-catch configuration with $1 \mathrm{MHz}$ probe, representative of well-bonded area (left) and of zone with inserts D1/D2 (right).

about the quality of the bond. In fact, analysis of FFTs shows amplitude variation of frequency components in the spectrum and the shift of some components with respect to the standard signal from a wellbonded region. In particular, on zone $\mathrm{A}$ and $\mathrm{D}$, where defects are in both layers, attenuation of the main frequency component is higher than on zone $\mathrm{B}$ and $\mathrm{C}$, respectively, with single layer disbond. Besides the attenuation of the main frequency component at about $0.85 \mathrm{MHz}$, there is a new component at lower frequency $(0.7 \mathrm{MHz})$.

Signals acquired with the $2 \mathrm{MHz}$ probe showed attenuation of the main frequency component (centered at about $1.1 \mathrm{MHz}$ ) on disbonded

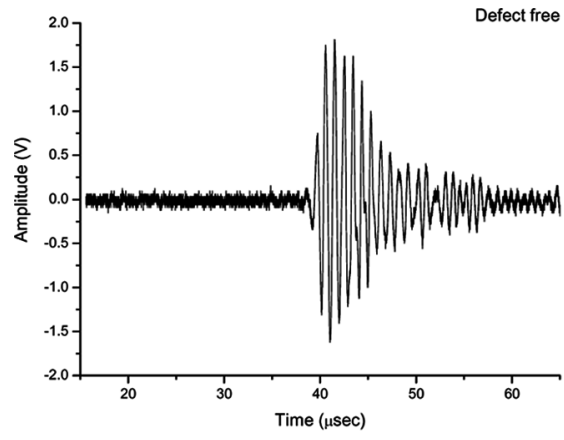

(a)

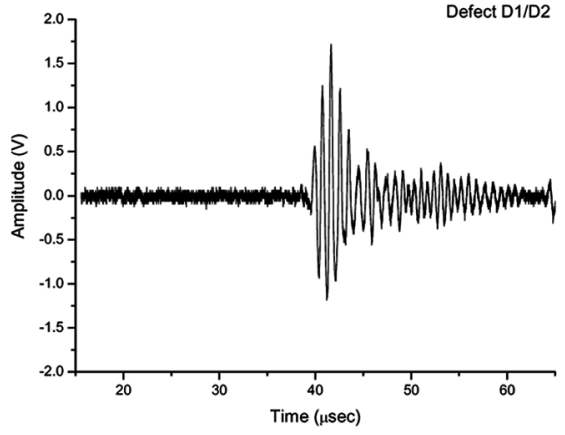

(b)

FIGURE 5 Time domain waveforms acquired in Sample \#1 in pitch-catch configuration with $2 \mathrm{MHz}$ probe, representative of well-bonded area (left) and of zone with inserts D1/D2 (right). 


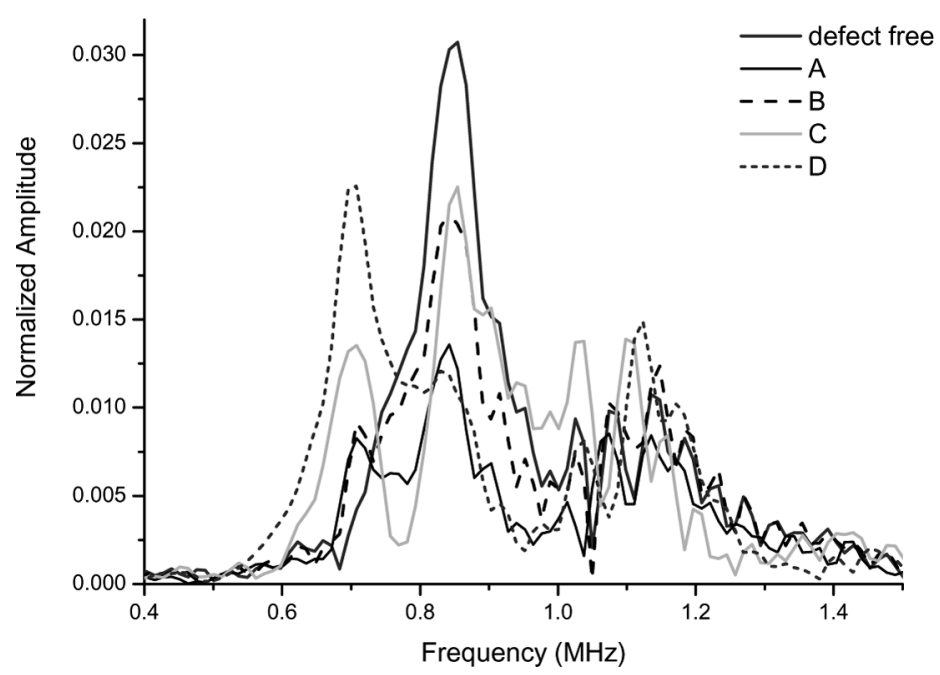

FIGURE 6 Normalized amplitude spectrum of signals, acquired in Sample \#1 in pitch-catch configuration with $1 \mathrm{MHz}$ probe, representative of wellbonded area and of zone with inserts (A1/A2, B, C, D1/D2).

areas and again between defects A and B, and B and C. Figure 7 shows the FFT of signals acquired in a well-bonded region (between defects $\mathrm{C}$ and D) and at defects A1/A2, B, C, and D1/D2. Only signals acquired at defects $\mathrm{A}$ and $\mathrm{B}$ show a new frequency component (at about $1.45 \mathrm{MHz}$ ) besides the attenuation of the main frequency component.

Inspection in zones between defects $\mathrm{A}$ and $\mathrm{B}$, and $\mathrm{B}$ and $\mathrm{C}$ provided waveforms similar to those in disbonded regions, only between defects $\mathrm{C}$ and $\mathrm{D}$ the waveform became regular and achieved maximum amplitude. The presence and growth of additional frequency peaks in Sample \#1 should be related to a mode converted waveform. However, a trend between the defects in the sample and the FFTs cannot be determined since besides the inserts there are areas of disbonds, due to a non-uniform condition of the adhesive layer (as shown by C-Scan tests and verified after cutting the sample) that affects in different ways the waves propagating through it.

Time domain waveforms acquired in Sample \#2 with the $1 \mathrm{MHz}$ probe are shown in Figure 8 for a well-bonded area (left) and for the zone with inserts E1/E2 (right). Figure 9 shows the FFTs of signals from a well-bonded region (reference signal) and disbonds (E1/E2, F, $\mathrm{G}$, and $\mathrm{H} 1 / \mathrm{H} 2$ ). If compared with the FFT spectrum relative to the waveform acquired in well-bonded areas in Sample \#1 with the same probe $(1 \mathrm{MHz}$ ) (see Figure 6), the results look quite different. In fact, 


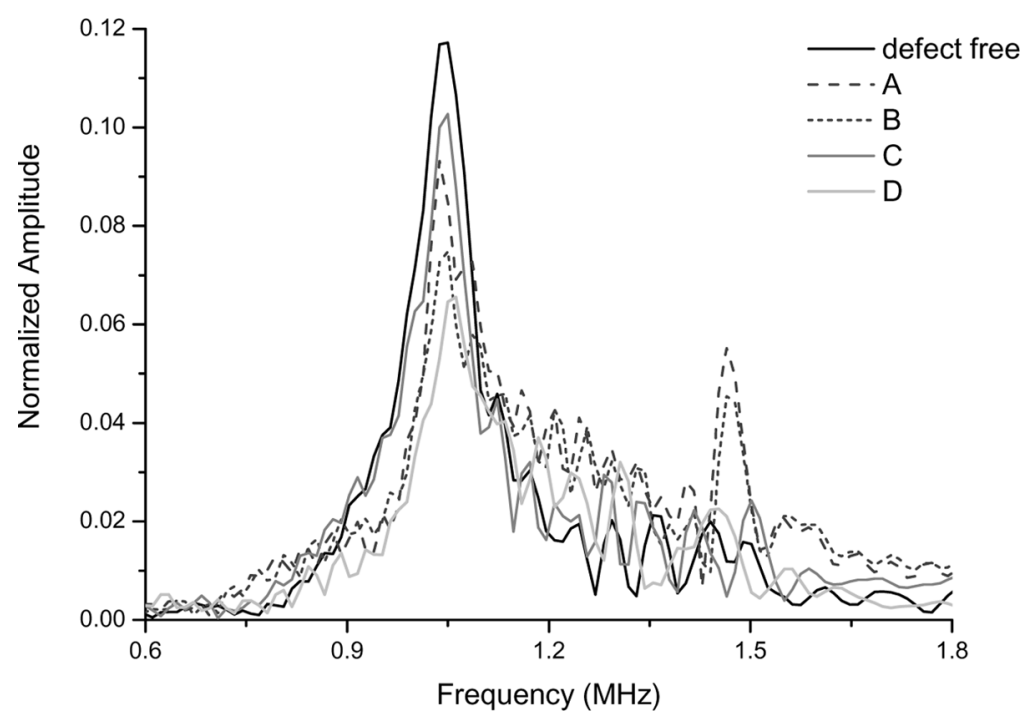

FIGURE 7 Normalized amplitude spectrum of signals, acquired in Sample \#1 in pitch-catch configuration with $2 \mathrm{MHz}$ probe, representative of wellbonded area and of zone with inserts (A1/A2, B, C, D1/D2).

Figure 9 shows a double peak FFT and frequency content that goes from 0.5 to $1 \mathrm{MHz}$, whereas, Figure 6 has a broader band FFT and frequencies from 0.7 to $1.3 \mathrm{MHz}$. The difference that causes the dissimilarity in the frequency spectrum is the different thickness of

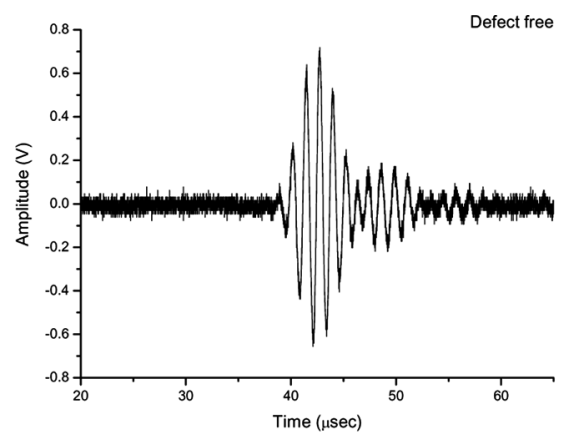

(a)

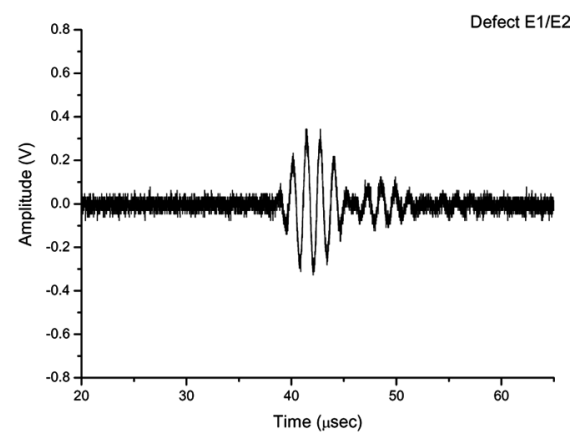

(b)

FIGURE 8 Time domain waveforms acquired in Sample \#2 in pitch-catch configuration with $1 \mathrm{MHz}$ probe, representative of well-bonded area (left) and of zone with inserts E1/E2 (right). 


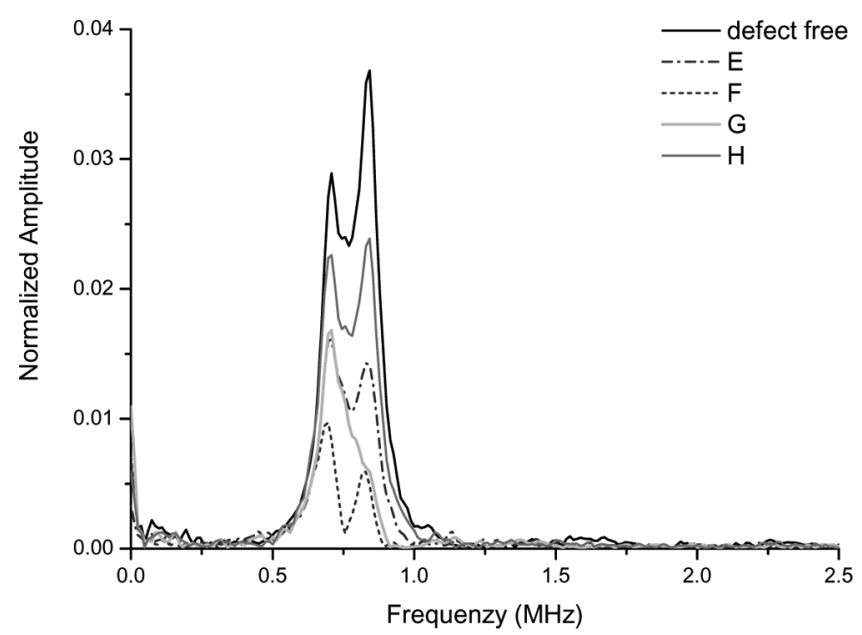

FIGURE 9 Normalized amplitude spectrum of signals, acquired in Sample \#2 in pitch-catch configuration with $1 \mathrm{MHz}$ probe, representative of wellbonded area and of zone with inserts (E1/E2, F, G, H1/H2).

aluminum and adhesive layers in the two samples (sequence is $2 / 0.3 / 2 / 0.3 / 2$ for $\# 1$ and $1 / 0.45 / 1 / 0.45 / 2$ for $\# 2$ ).

However, similarly to Sample \#1, attenuation of frequency components was related to status of disbonds. Outside disbonds, signals showed a regular and standard waveform, referring to a uniform quality of the multi-layer structure. The repeatability of signals acquired in defect-free regions in Sample \#2 was high, as the amplitude variation between different defect-free measurement points was within $\pm 7 \%$. Figure 10 shows three different waveforms representative of well-bonded areas.

Analysis of signals acquired in both samples with the $500 \mathrm{kHz}$ probe did not show significant differences in waveforms or frequency content along the direction of the scan.

\subsection{C-Scan Test}

This test was performed to obtain a precise mapping of disbonded areas. The amplitude of the longitudinal wave at normal incidence, acquired with a $1 \mathrm{MHz}$ probe as receiver, was plotted as a function of the $\mathrm{x}$, y position during scanning at $5 \mathrm{~mm}$ steps. Inspection was limited to 2 and $4 \mathrm{~cm}$ along the $\mathrm{x}$-direction for Samples $\# 1$ and $\# 2$, respectively, in the central area of the samples. Figures 11(a) and 


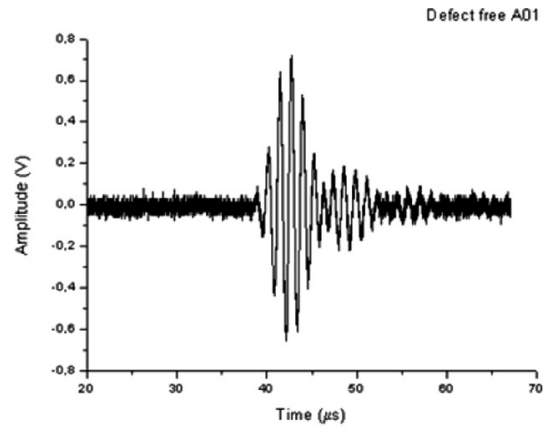

(a)

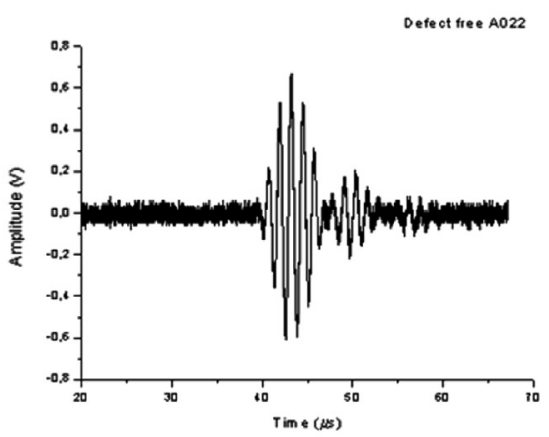

(b)

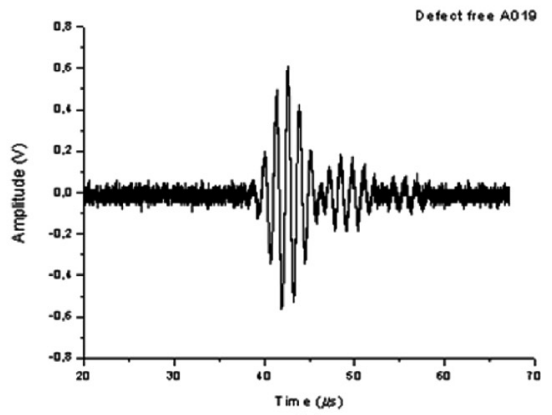

(c)

FIGURE 10 Time domain waveforms acquired in Sample \#2 in pitch-catch configuration with $1 \mathrm{MHz}$ probe, representative of well-bonded areas. Peakto-peak amplitude variation is within $\pm 7 \%$.

11(b) show the C-Scan maps, respectively, for Samples \#1 and \#2, produced by converting the normalized amplitudes to a grey-scale colormap. The lighter color in the map is for the maximum normalized amplitude and the darker colored area relates to the highest loss in signal amplitude (0.15 for Sample \#1 and 0.067 for Sample \#2), as the ultrasonic wave encounters a different density medium in the bulk of the multi-layer, due to the discontinuities that cause either partial or total reflection of the wave. The position of the inserts is indicated with dotted lines above the maps in Figures 11(a) and 11(b).

C-Scan maps gave a clear indication of disbonded areas in both samples, even though a smaller step size would have been necessary to plot the precise size of the defects. In Sample \#1, besides the introduced inserts, other disbonded areas were visible, confirming the previous results obtained with the L-Scan configuration, probably due to non-evacuation of air in the adhesive, trapped during manufacturing. 


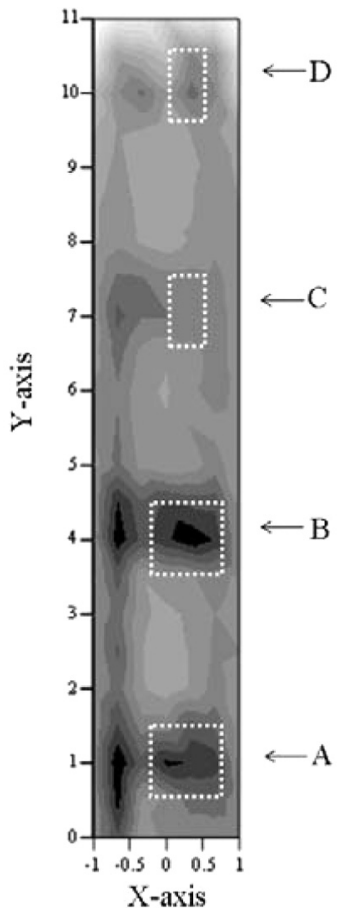

(a)

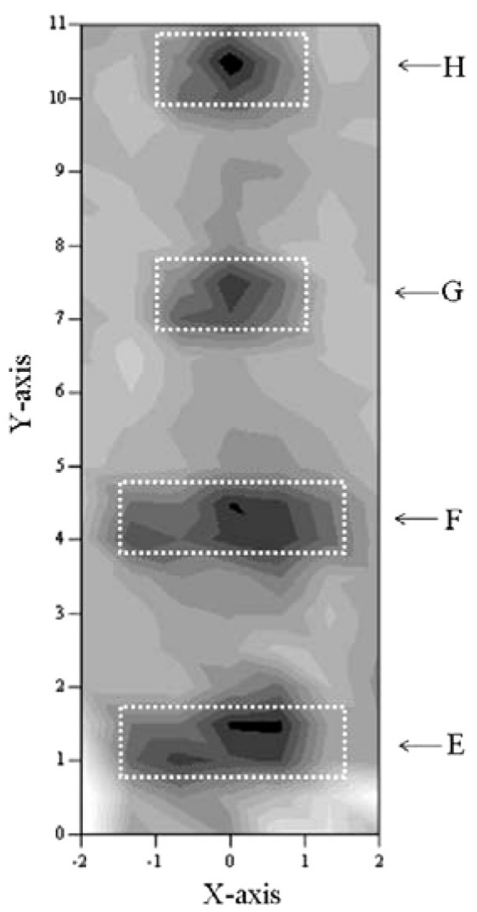

(b)

FIGURE 11 Normalized amplitudes of the longitudinal wave at normal incidence, presented as a grey-scale C-scan map for (a) Sample \#1 and (b) \#2 (scale in centimeters). Color code goes from white (relative to 1) to black (0.15 for Sample \#1 and 0.067 for Sample \#2). Real position of inserts is indicated by dotted lines.

After the UT measurements, Sample \#1 was cut along lines 11 and 22, indicated in Figure 12, to verify the bond quality plus the position of inserts A1/A2 and D1/D2 in the cross-section, since the C-Scan map could be misinterpreted. Macrographs of the central area in the cross-section, taken with a high resolution camera, are shown in Figures 13 and 14. The position at the two interfaces of inserts A1 and A2 is indicated in Figure 13, where other discontinuities in the adhesive layer are also visible, as expected from the C-Scan map. Inserts D1 and D2 are indicated in Figure 14 where a $2 \mathrm{~mm}$ shift is visible between the two, maybe due to the phase of placement of the Al layers, plus an extended separation at the adhesive/aluminum interface next to D2. 


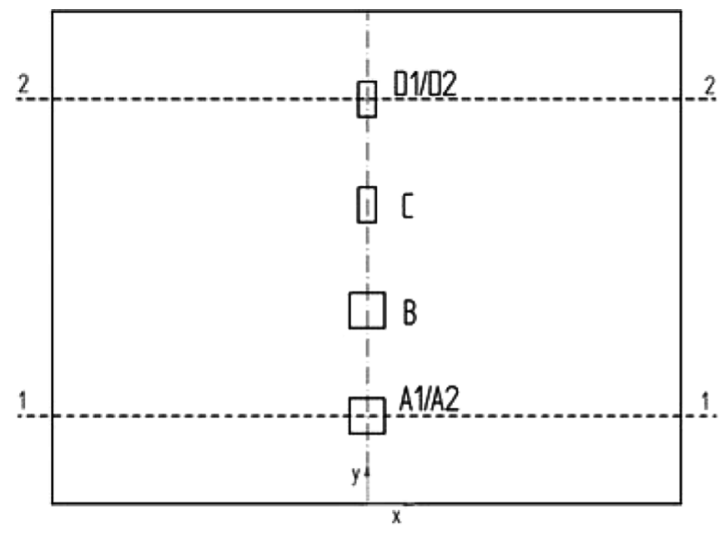

FIGURE 12 Indication of position of lines 11 and 22 along which Sample \#1 was cut.

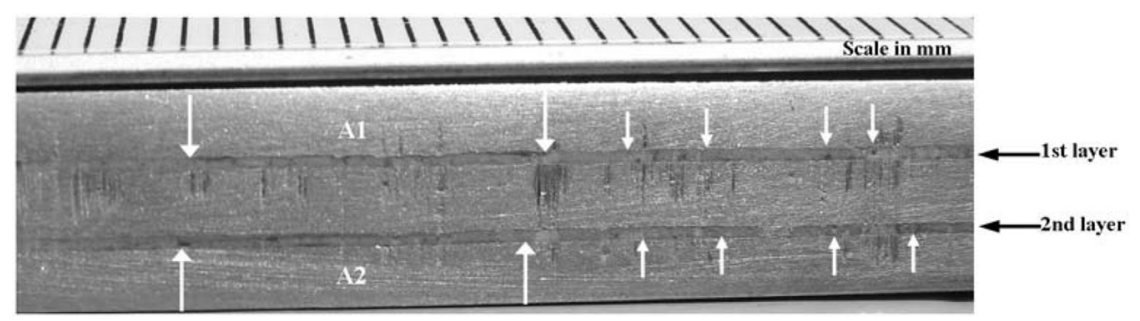

FIGURE 13 Macrograph of the central area in Sample \#1 after cutting along line 11. Location of inserts A1 and A2 at the two interfaces is indicated. Other discontinuities in the adhesive are pointed out.

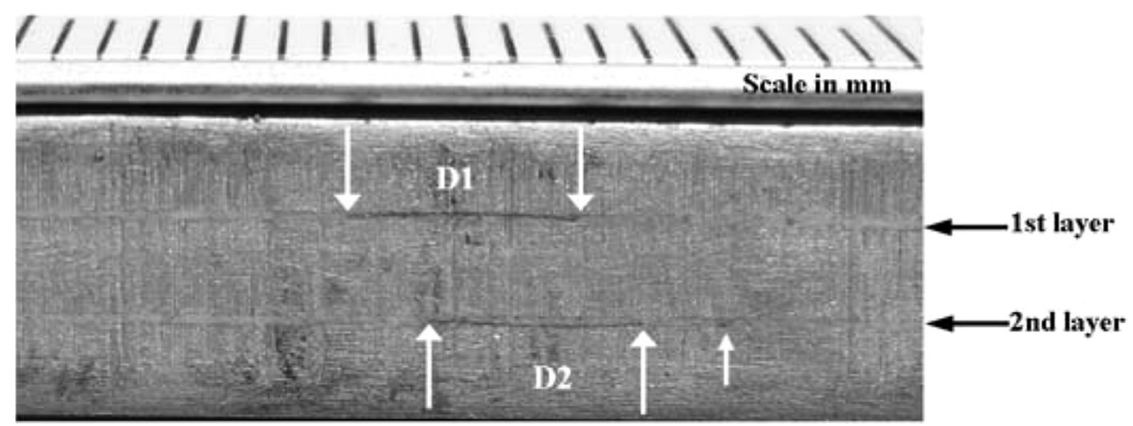

FIGURE 14 Macrograph of the central area in Sample \#1 after cutting along line 22. Location of inserts D1 and D2 at the two interfaces is indicated. A 2-mm shift between D1 and D2 is visible, plus an extended disbond next to D2. 
The C-Scan map of Sample \#1 allowed us to define the disbonded areas and to justify the considerations previously made with the L-Scan. Zones between defects $\mathrm{A}$ and $\mathrm{B}$, and $\mathrm{B}$ and $\mathrm{C}$ also show discontinuities; between $\mathrm{B}$ and $\mathrm{C}$ the disbond is less extended as the smaller attenuation was showing.

The C-Scan map of Sample \#2 confirmed results from the L-Scan plus it provided 2-D sizing of disbonds even though the contour line was not well defined due to the $5 \mathrm{~mm}$ scan step. C-Scan through-transmission configuration allowed us to confirm results obtained with the L-Scan configuration, even though to inspect a smaller area more time was required.

\section{CONCLUSION}

Laser-based ultrasonic inspection of bonding interfaces in multi-layer $\mathrm{Al}$ structures has been investigated. Since multi-layer structures are widely used in many industries, especially aerospace, for their structural efficiency, the integrity and reliability of the bonds are extremely important to the quality of the product. A practical inspection procedure using a laser-based ultrasonic method and a L-Scan pitch-catch configuration has been used to assess disbonded conditions at the adhesive/aluminum interface. Detection of adhesion defects was quickly achieved, thus, showing the inherent advantage for the inspection of large structures, even though L-Scan makes it impossible to detect the position of disbonds along a direction different from that of scanning.

It is evident that the method is effective in evaluating the quality of bonding interfaces since results are in agreement with those obtained by a C-Scan in the through-transmission configuration that was more appropriate to determine the $2 \mathrm{D}$ dimension but more time consuming, plus it required access on both sides.

\section{REFERENCES}

[1] Higgins, A., International Journal of Adhesion \& Adhesives 20, 367-376 (2000).

[2] Baker, A., Duttou, S., and Kelly, D., Composite materials for aircraft structures, (American Institute of Aeronautics \& Astronautics, Reston, VA, 2004)

[3] Wacker, G., Brügge, D., Fach, K., and Franzelius, W., Proc. of the Institution of Mechanical Engineers, Part M: Journal of Engineering for the Maritime Environment 218, 217-226 (2004).

[4] Shull, P. J., Nondestructive Evaluation: Theory, Techniques, and Applications, (Marcel Dekker, Inc., New York, 2002). 
[5] Menaka, M., Bagavathiappan, S., Venkatraman, B., Jayakumar, T., and Baldev, R., Insight 48, 606-612 (2006).

[6] Brotherhood, C. J., Drinkwater, B. W., and Dixon, S., Ultrasonics 41, 521-529 (2003).

[7] Lowe, M. J. S. and Cawley, P., Journal of Nondestructive Evaluation 13, 185-200 (1994).

[8] Bhardwaj, M. C., Encyclopedia of Smart Materials, A. Biderman, Ed. (John Wiley \& Sons, New York, 2001).

[9] Blomme, E., Bulcaen, D., and Declercq, F., Ultrasonics 40, 153-157 (2002).

[10] Scruby, C. B. and Drain, L. E., Laser Ultrasonics: Techniques and Applications, (Adam Hilger, Bristol, UK, 1990).

[11] Kenderian, S., Djordjevic, B. B., Cerniglia, D., and Garcia, G., Insight 48, 336-341 (2006).

[12] Kenderian, S., Cerniglia, D., Djordjevic, B. B., and Green, R. E., Jr. Research in Nondestructive Evaluation 16, 195 (2005).

[13] Schindel, D. W., Ultrasonics 37, 185-200 (1999).

[14] Hsu, D. K., Ultrasonics 44, 1019-1024 (2006).

[15] Heller, K., Jacobs, L. J., and Qu, J., NDT\&E International 33, 555-563 (2000).

[16] Rose, J. L., Ultrasonic Waves in Solid Media, (Cambridge University Press, New York, 2004).

[17] Dalton, R. P., Cawley, P., and Lowe, M. J. S., Journal of Nondestructive Evaluation 20, 29-46 (2001).

[18] Viktorov, I. A., Rayleigh and Lamb Waves, (Plenum Press, New York, 1967).

[19] Davies, S. J., Edwards, C., Taylor, G. S., and Palmer, S. B., Applied Physics 26, 329-348 (1992).

[20] Mi, B. and Ume, I. C., Journal of Nondestructive Evaluation 21, 23-33 (2002).

[21] Murray, T. W., Deaton, J. B., Jr., and Wagner, J. W., Ultrasonics 34, 69-77 (1996).

[22] Cosenza, C., Kenderian, S., Djordjevic, B. B., Green, R. E., Jr., and Pasta, A., IEEE Trans. on UFFC 54, 147-156 (2007). 